\title{
Úlcera en lengua causada por remanentes radiculares. Reporte de caso
}

\author{
A tongue ulcer caused by radicular remanents. Case report \\ Rocío Miranda ${ }^{1, a, d, e}$, Sofía Cuba ${ }^{2, b, f, h}, A$ gustín Gamarra ${ }^{2, c, g}$

\section{RESUMEN}

Un problema frecuente de las personas que se atienden en el primer nivel de atención son las lesiones orales, por lo que los médicos en general y en particular los de atención primaria, deben estar capacitados en reconocerlas. Muchas causas pueden dar como resultado una úlcera en la mucosa oral, por lo que el diagnóstico diferencial puede ser difícil. Se presenta el caso de una mujer de 47 años, que acudió a un centro de atención primaria por presentar una lesión ulcerosa localizada en la parte inferior de la lengua, que resultó ser secundario a un problema dental.

PALABRAS CLAVE: Boca, úlceras bucales, atención primaria en salud. (Fuente: DeCS BIREME).

\section{SUMMARY}

A common medical problem that primary care physicians encounter in daily practice are oral lesions, for which proper training to identify the possible causes is necessary. Many potential etiologies may cause an oral ulcer, making the differential diagnosis difficult. We present the case of a 47 year-old woman who presented at a primary care center with an ulcer located in the inferior part of the tongue that resulted from a dental problem.

KEYWORDS: Mouth, oral ulcer, primary health care. (Source: MeSH NLM).

\section{INTRODUCCIÓN}

Según el Ministerio de Salud (MINSA), el 92\% de la población ha tenido o tiene algún problema de salud oral (1). El médico de atención primaria debe estar capacitado en reconocer aquellas que se autolimitarán o serán de resolución en el nivel y de derivar las que requieran una aproximación diferente.

La mucosa oral soporta numerosos traumas de diverso origen. En ella se pueden manifestar patologías de origen traumático, infeccioso, neoplásico, entre otros (2). Una de las lesiones más frecuentes es la úlcera, que puede tener causas diversas, siendo necesario determinar con exactitud el origen de la lesión para su adecuado tratamiento.

En esta comunicación, presentamos el caso de una mujer adulta con una úlcera en la mucosa oral, quien fue evaluada por distintas especialidades antes de llegar al diagnóstico final.

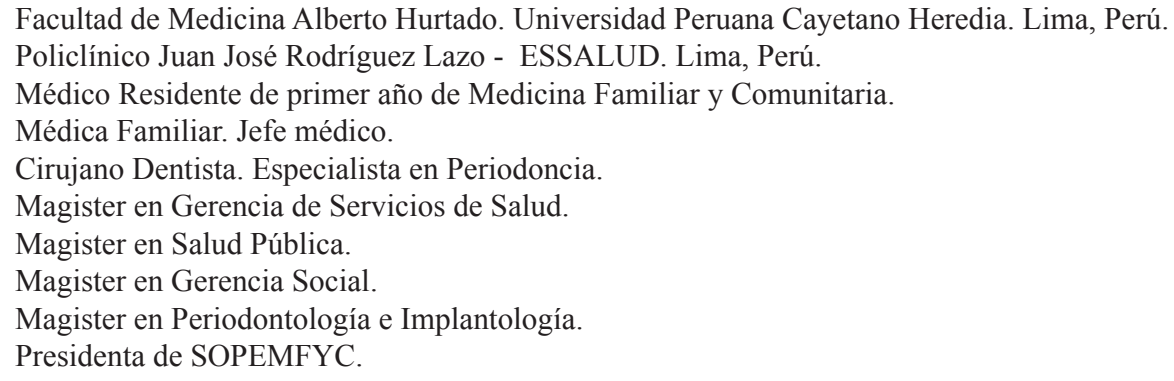




\section{Caso clínico}

Mujer de 47 años, casada, natural de Cusco, procedente de Lima, acudió al servicio de Medicina Familiar en un Centro de Atención Primaria de ESSALUD, por presentar lesión ulcerosa de bordes definidos localizada en el dorso de la lengua, sin variación en el tamaño, de dos meses de evolución (Figura 1).

La paciente había sido evaluada previamente por las especialidades de Cirugía y Otorrinolaringología, practicándosele una biopsia que fue informada como tejido necrótico. Al examen se apreció en el maxilar inferior izquierdo, dos remanentes radiculares de superficie cortante que coincidían con la zona del lesión en la cara inferior de la lengua. El resto de examen físico fue normal. Se envió a la paciente al servicio de Odontología, donde se le realizó exodoncia de los remanentes radiculares.

Fue evaluada un mes después, apreciándose que la lesión había remitido, mostrando una superficie lingual normal (Figura 2).

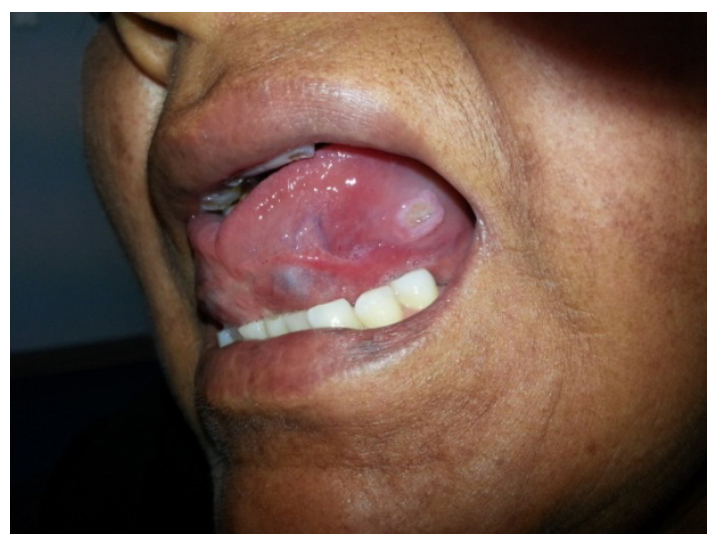

Figura 1. Lesión crónica en cavidad oral de características ulcerosas en la cara inferior de la lengua.

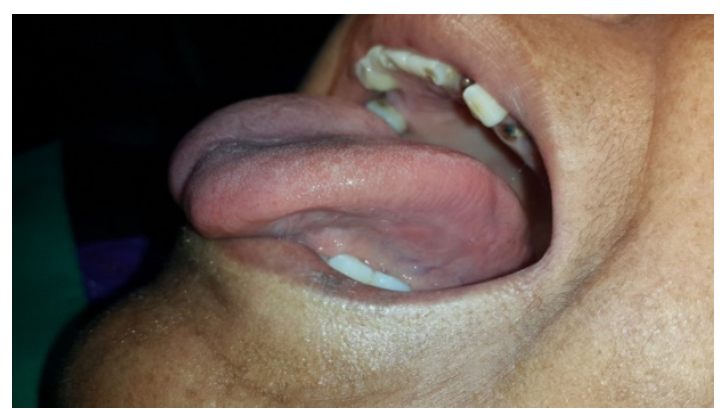

Figura 2. Un mes después de la exodoncia de los remanentes radiculares.

\section{DISCUSIÓN}

Una úlcera es definida como una solución de continuidad de la superficie epitelial que llega a comprometer todos los estratos epiteliales. Muchas causas pueden dar como resultado una úlcera en la mucosa, por lo que el diagnóstico diferencial puede ser difícil. Se pueden clasificar en (3): a) Úlcera primaria, aquella que aparece sin otra lesión como antecesora, por ejemplo, úlcera traumática, afta; b) Úlcera secundaria, aquella que resulta de la ruptura de una vesícula, dejando un área ya sea erosionada o francamente ulcerada, por ejemplo, herpes.

Sin embargo, la clasificación etiológica es más útil, porque al plantear la causa nos orienta en el tratamiento: a) Úlcera traumática, secundaria a sustancias cáusticas, radiación, medicamentos, quemaduras, prótesis dentales, mal cepillado, etc; b) Úlcera neoplásica: carcinoma espinocelular, adenocarcinoma, sarcoma, linfoma, etc; c) Úlcera infecciosa: gingivitis, sífilis, tuberculosis, herpes, etc; d) Úlcera inmunológica: pénfigo, penfigoide, síndrome de Behcet, etc; y e) Úlcera por enfermedad sistémica: déficit de ácido fólico, hierro, vitamina B12, diabetes, etc.

La úlcera traumática puede ser causada por prótesis, cepillado inadecuado, borde filoso de una pieza dentaria fracturada o cariada e irritantes externos como medicamentos, ácidos o álcalis (4). Las localizaciones más frecuentes son: borde lateral de la lengua, cara interna de la mejilla, labios y ocasionalmente en el paladar. Aunque la mayoría de veces la curación es rápida, eventualmente existen úlceras que persisten largo tiempo sin cicatrizar. Esto es importante porque pueden llegar a tener gran similitud con las lesiones producidas por los carcinomas y, a veces, son sometidas a biopsias repetidas para tratar de establecer el diagnóstico de neoplasia (5).

Como parte del diagnóstico diferencial, también se deben considerar a las lesiones autoinducidas, producidas por algún hábito como el de morderse el labio o la mejilla. Aquí se encuentran además las lesiones producidas durante un ataque de epilepsia (6).

Los agentes externos como medicamentos (ácido acetilsalicílico), ácidos, álcalis, enjuagatorios en soluciones alcohólicas, también producen lesiones ulcerativas o erosiones cuando son aplicados directamente sobre la mucosa. Muchos producen una necrosis epitelial, que clínicamente aparece como una lesión plana, de bordes irregulares y cubiertos por 
un epitelio necrótico que al ser removido deja una superficie sangrante (7). El ácido acetilsalicílico es el agente que con más frecuencia produce estas lesiones. Los usuarios de drogas, por ejemplo cocainómanos, también las pueden presentar (8). Pueden provocarse quemaduras térmicas cuando el paciente ingiere alimentos o bebidas muy calientes (9).

La úlcera traumática causada por irritación protésica es una lesión que se desarrolla generalmente pocos días después de la inserción de prótesis nuevas, como resultado de una confección inadecuada de ellas (10).

En un estudio epidemiológico realizado por el MINSA en el 2003 sobre enfermedades orales, se encontró que el $92 \%$ de la población tiene o ha tenido caries (1), y sabiendo que provoca desmineralización de las piezas dentarias llevándolas a la posterior fractura y convirtiéndolas en remanentes radiculares, debemos tener en cuenta esta información al observar lesiones orales en los pacientes.

Como se ha ido revisando, el aspecto clínico de la mayoría de lesiones depende de la gravedad del daño tisular. La mejor manera de identificar estas lesiones es una buena anamnesis.

Se debe considerar así mismo, que si la causa se hace crónica, las características de la lesión se modifican, debiendo plantearse otras causas en el diagnóstico diferencial. En este caso, el siguiente paso será la realización de una biopsia para su estudio anatomopatológico (9).

En la cavidad bucal debemos detectar las lesiones potencialmente malignas para reducir la capacidad de transformación, para ello debemos conocerlas, diagnosticarlas y tratarlas de forma oportuna $(11,12)$.

En este caso, a pesar del resultado negativo de la biopsia, continuaron buscando una causa maligna de la úlcera. Si bien es cierto, en toda lesión de este tipo hay que considerar como un diagnóstico diferencial a las lesiones neoplásicas, es importante pensar en el contexto del ámbito donde nos encontramos. En el primer nivel de atención se dispone de otras herramientas que puedan ayudar a la mejor aproximación en el diagnóstico: la continuidad con el paciente, el conocimiento de su contexto y el trabajo en equipo.

Fueron estos tres factores los que ayudaron a resolver el problema de la paciente evitando nuevas intervenciones que pudieran generar complicaciones, gastos innecesarios y angustia en la persona. Barbara Starfield (13) de Johns Hopkins University, sostenía que mientras un paciente se mantiene más en el seno del primer nivel, a cargo de un grupo de profesionales capacitados, mejores serán los resultados para su salud.

\section{Declaración de financiamiento y de conflictos de interés:}

Los autores declaran haber realizado este reporte con recursos propios y que no tienen conflictos de intereses.

\section{Créditos de autoría:}

RM: Participó en la revisión de caso y de la bibliografía; SC y AG: en la selección de caso y la revisión de la bibliografía.

\section{Correspondencia:}

Rocio Miranda

meromi295@hotmail.com

\section{REFERENCIAS BIBLIOGRÁFICAS}

1. Domínguez G, Arellano L, Velezmoro V. Estudio epidemiológico de caries dental en el Perú. Revista del Colegio Odontológico del Perú. 2003; 8:12.

2. Salinas Y, Millán R, León J. Lesiones traumáticas en cavidad oral. Acta Odontológica Venezolana. 2008; 46: 1-6.

3. Quinteros, I. Lesiones ulcerativas en mucosa oral. Tesis de grado. Santiago de Chile, Chile. Facultad de Odontología, Universidad de Chile. 1983. 1-65 pp.

4. Cawson RA. Oral ulceration: Clinical aspects. Oral Surgery, Oral Medicine, Oral Pathology. 1972; 33: 912-921.

5. Wanda C, Gonsalves M, Angela C. Common oral lessions. American Family Physician. 2007; 75: 501507.

6. Acosta M, Francisco H. Alteraciones bucales encontradas en pacientes epilépticos. Revista ADM. 1998; 55: 29-33.

7. Najjar T. Harmful effects of aspirin compound. Oral Surgery, Oral Medicine, Oral Pathology. 1977; 44: 64-70.

8. Dello Russo N, Temple H. Cocaine effects on gingiva. Journal American Dental Associated. 1982; 104: 1316.

9. Salinas J, Ronald E. Lesiones traumáticas: Conducta odontológica. Acta Odontológica Venezolana. 2013; 
46: 543-546.

10. Díaz M, Martell Y, Zamora J. Afecciones de la mucosa oral encontradas en pacientes geriátricos portadores de prótesis estomatológicas. Revista Cubana de Estomatología. 2007; 44: 1-4

11. Carnevale S, Bassan N, Roy O. Detección de lesiones bucales en una población geriátrica. Buenos Aires: Actualizaciones odontológicas; 2009.p. 1-4.

12. Aguas S, Lanfranchi H. Lesiones premalignas o cancerizables de la cavidad oral. Revista de la Facultad de Odontología. 2004; 19: 21-30.
13. Starfield B. Atención primaria. Equilibrio entre necesidades de salud, servicios y tecnología. Barcelona: Masson S.A.; 2004.p.153.

Recibido: 02/02/2014

Aceptado: 27/08/2014 